\title{
Early Palliative Referral Reduces the Odds of Receiving Chemotherapy During the Last 30 Days of Life, a Single Center Experience From Windsor Essex, Ontario, Canada.
}

Indryas Woldie ( $\square$ indryas.woldie@wrh.on.ca )

Windsor Regional Hospital

Tarek Elfiki

Windsor Regional Hospital

Swati Kulkarni

Windsor Regional Hospital

Colvin Springer

Windsor Regional Hospital

Eric McArthur

London Health Sciences Centre

Nicole Freeman

Windsor Regional Hospital

\section{Research Article}

Keywords: Chemotherapy, palliative care, end of life

Posted Date: September 7th, 2021

DOl: https://doi.org/10.21203/rs.3.rs-842473/v1

License: (c) (i) This work is licensed under a Creative Commons Attribution 4.0 International License.

Read Full License 


\section{Abstract}

Background: Chemotherapy use closer to the end of life is becoming an important issue in cancer care. There are now multiple studies and local reviews addressing this issue. Understanding the practice locally will give valuable insight and opportunity for improvement.

Methods: The study is a retrospective chart review of patients on chemotherapy at the Windsor Regional Cancer Center who died between April $1^{\text {st }}, 2016$ to December $31^{\text {st }}, 2018$. Information on demographics, type of cancer, type, intent and route of chemotherapy, line of chemotherapy, referral to hospice and palliative care services was collected.

Results: A total of 681 patients on chemotherapy died between April $1^{\text {st }}, 2016$ to Dec $13^{\text {th }}, 2018$. Of these, $119(17.4 \%)$ passed away within 30 days following chemotherapy. Chemotherapy was parenteral (Intravenous and Subcutaneous) for the majority (75.2\%) of the patients. Most (66.4\%) of the patients died of disease progression. Intent for chemotherapy was palliative in $85 \%$ of patients, adjuvant/neoadjuvant in $6.6 \%$ and curative in $8.4 \%$ of the patients. Chemotherapy was $1^{\text {st }}, 2^{\text {nd }}, 3^{\text {rd }}$ line or more in $67.4 \%, 21.3 \%$ and $11.3 \%$ of the patients respectively. The type of chemotherapy was conventional in $74.3 \%$ of patients and targeted/immunotherapy in $25.7 \%$ of patients.

Of the variables studied, lack of palliative referral and having lung cancer or melanoma were significantly associated with higher risk of getting chemotherapy within the last four weeks of life. The odds of getting chemotherapy in within the last four weeks of life is $0.35,95 \% \mathrm{Cl}(0.24-0.53), \mathrm{P}<0.001$ for those who were referred to palliative care. On the other hand, the odds of getting chemotherapy was $4.18,95 \% \mathrm{Cl}(1.17-$ 13.71), $\mathrm{P}=0.037$ and $2.21,95 \% \mathrm{Cl}(1.24-4.01), \mathrm{P}=0.037$ for those with melanoma and lung cancer respectively.

Conclusion: Administration of chemotherapy within the last 30 days of life could cause unnecessary suffering to patients and cost to society. Early referral to palliative care was significantly associated with reduced risk of getting chemotherapy within the last 30 days of life in this study. Prospective study is recommended to further investigate the role of early palliative referral on use of chemotherapy during the last 30 days of life.

\section{Introduction:}

Chemotherapy use closer to the end of life is becoming an important issue in cancer care. It is a complex decision-making process that involves patient, family, oncologist and other members of the cancer care team. Chemotherapy at the end of life could decrease survival and increase health care utilization according to some studies (1-4).

Although the role of effective communication and involvement of palliative care has been emphasized as a solution, studies have shown considerable variation in transition from active therapy to end of life care (5). A study from the United States showed many oncologists were reluctant to prescribe chemotherapy 
at the end of life. On the other hand, a patient's decision depends on the clarity of information s/he receive (6).

A study from France showed patients who died in for profit hospitals, comprehensive cancer centers and centers without palliative care had greater than average use of chemotherapy near the end of life (7). Very high rates of chemotherapy in the last 30 days of life were noticed in centers with no or limited palliative care services as well (8).

Another critical issue isthe accuracy of oncologists' predictions of survival. A study found out that oncologists made accurate predictions of survival in around a third of patients (9). Finally, the role of chemotherapy in palliating symptoms should also be taken into account. This is especially true with the newer targeted and immunotherapeutic agents that are convenient to administer, could work quickly to palliate symptoms, and could be used more and more in the future (10).

This retrospective study will describe the rate of chemotherapy administered within the last 30 days of life and associated factors at a regional cancer center in Ontario, Canada.

\section{Methods:}

The study is a retrospective chart review of 681 patients on chemotherapy at the Windsor Regional Cancer Center who died between April 1st, 2016 and December 31st, 2018 per hospital records.

Chemotherapy was defined as conventional/cytotoxic/, targeted therapy and immunotherapy based on the last chemotherapy regimen preceding patient's death. Patients receiving hormonal therapy as the only cancer treatment were excluded. Information on demographics, type of cancer, type, intent and route of chemotherapy, line of chemotherapy and referral to palliative care services was collected. These variables were selected during the inception of the study by the investigators based on their clinical judgment. Ethical clearance was obtained from the ethics review board of the Windsor Regional Hospital.

Continuous variables were presented as median (interquartile range) and categorical variables were presented as frequency (percentage). Univariable associations for factors of interest were estimated using odds ratios and their associated $95 \%$ confidence intervals, obtained from logistic regression models. Two-sided p-values $<0.05$ were considered statistically significant.

\section{Results:}

A total of 681 patients on chemotherapy died between April 1st, 2016 to Dec 31st, 2018. Of these, 119 (17.4\%) passed away within 30 days following chemotherapy, with a median of 17 days (range 1-30 days). Of those who died within 30 days of chemotherapy: $55.5 \%$ were male with a median age of 69 years. Intent for chemotherapy was palliative in $89.9 \%$ of patients, curative in $6.7 \%$ of the patients and adjuvant/neoadjuvant in $3.4 \%$. Chemotherapy was $1 \mathrm{st}$, 2 nd, 3 rd line or more in $70.6 \%, 16 \%$ and $13.4 \%$ of the patients respectively. The type of chemotherapy was conventional in $73.9 \%$ of the patients and targeted/immunotherapy in $26.1 \%$ of patients. Oral chemotherapy was used in a third $(29.4 \%)$ of the 
patients, while the rest was parenteral (Intravenous or Subcutaneous). The three most common types of cancer in those patients who died within 30 days following chemotherapy were lung (30.3\%), hematologic (22.7\%) and gastro-intestinal (18.5\%). Only $44.5 \%$ of the patients who died within 30 days of chemotherapy were referred to palliative care. [Table 1]

Almost half (49\%) of the patients passed away in the hospital, whereas $15.5 \%$ passed away in hospice and $15.5 \%$ passed away at home. Place of death was unknown for $20 \%$ of patients.

Of the variables evaluated, referral to palliative care and type of cancer were significantly associated with the likelihood of receiving chemotherapy in the last 30 days of life. Patients who were referred to palliative care were less likely to receive chemotherapy within the last 30 days of their life [OR $0.35,95 \%$ $\mathrm{Cl}(0.24-0.53), \mathrm{P}<0.001]$. In addition, the earlier the referral to palliative care, the lesser the odds of receiving chemotherapy within the last 30 days of life. With respect to cancer type, compared to gastrointestinal cancer, lung cancer $[2.21,95 \% \mathrm{Cl}(1.24-4.01), \mathrm{P}=0.037]$ and melanoma $[4.18,95 \% \mathrm{Cl}$ (1.17-13.71), $P=0.037$ ] were significantly associated with higher odds of receiving chemotherapy within the last 30 days of life. [Table 2]

\section{Discussion:}

The study has several limitations including retrospective design, lack of details on circumstances of around the decision to stop chemotherapy, lack of information on insurance which might affect access to oral chemotherapy and relatively small sample size. Despite its limitations, it shed some light on local practices with respect to chemotherapy use around end of life.

In our study, $17.4 \%$ of patients passed away within 30 days of chemotherapy which is similar to other studies from USA, France and Taiwan, but higher than a study from Switzerland that showed only $11.7 \%$ of patients received chemotherapy in the last 4 weeks of their life. $(7,11-13)$

However, our results are much lower than the data from Uganda and Egypt that showed $45 \%$ and $56 \%$ of patients in these countries, respectively, received chemotherapy in their last month of life. $(8,14) \mathrm{A}$ possible explanation could be limited availability of palliative care services although cultural differences in end-of-life care could also play a role. Our study also showed patients who were referred to palliative care were less likely to receive chemotherapy within the last 30 days of their life. This is further supported by a study from France that showed young individuals, those treated in comprehensive cancer centers or high-volume centers without palliative care units were the most likely to receive chemotherapy near the end of life. (7) Another multicenter study from the USA showed quality of life near death is not improved and can be harmed by chemotherapy near death even in patients with good performance status. $(15,16)$

This is an interesting paradox particularly in low resource areas with limited palliative care service resulting in the use of more expensive and potentially harmful chemotherapy with futile outcome around the end of life. This suggests the need to build palliative care services hand in hand with active therapy in cancer treatment centers. 
Our study showed only referral to palliative care and type of cancer were significantly associated with the likelihood of receiving chemotherapy in the last 30 days of life. Patients who are not referred to palliative care and those with either lung cancer or melanoma were more likely to receive chemotherapy within the last 30 days of their life. A study in Ontario showed younger age, male gender, hematologic malignancies and breast cancer were all associated with more aggressive care, which among others includes last dose of chemotherapy within the last 14 days of life. (17) Smaller sample size in our study might be the reason for the lack of statistically significant associations for some of the variables.

A study from New York showed chemotherapy in the last 30 days of life was associated with an increased rate of hospital admissions, emergency department visits, death in the hospital, fewer days in hospice care and a more than $50 \%$ increase in patient out of pocket costs for care. Another study from Taiwan showed similar findings. $(3,15)$ Our study also showed almost half of the patients who received chemotherapy within the last 30 days of life died in the hospital.

Finally, the benefits of early palliative referral cannot be overemphasized as there is evidence for improved quality of life and prolonged survival in patients referred to palliative care. (18) It is also reasonable to expect more overlap between active therapy and palliative care as more targeted and immuno-therapies become available. This shift from conventional chemotherapy, particularly in advanced disease, could result in less toxicity and better efficacy. (10)

\section{Conclusions:}

The use of chemotherapy in the last 30 days of life could result in inconvenience and unnecessary cost to patients and the health care system. Early palliative referral is associated with less use of chemotherapy around the end of life in this study and needs further prospective investigation. Conducting continuous audit on end-of-life care are also recommended.

\section{Declarations:}

\section{Ethics approval and consent to participate:}

Ethical clearance was obtained from the ethics review board (REB) of the Windsor Regional Hospital. Consent to participate was waived by the REB as the study is retrospective chart review and poses minimal risk to participants.

\section{Consent for publication:}

Not applicable

\section{Availability of data and materials:}

The datasets used and/or analyzed during the current study available from the corresponding author on reasonable request. 
Authors contribution:

IW, TE and SK contributed in the conception of the study. IW, TE, SK, CS, NF contributed in data acquisition. IW, EM and NF contributed in data analysis and interpretation of the results. IW and EM drafted the manuscript. All authors critically reviewed the manuscript and approved with the final version.

\section{Competing Interests:}

No relevant conflict of interest for all authors.

\section{Funding:}

this research received no specific grant from any funding agency in the public, commercial, or not-forprofit sectors.

\section{Acknowledgement:}

we would like to acknowledge Windsor Regional Hospital administration decision support team for facilitating data acquisition.

\section{References:}

1. Zhu Y, Tang K, Zhao F, Zang Y, Wang X, Li Z, et al. End-of-life chemotherapy is associated with poor survival and aggressive care in patients with small cell lung cancer. J Cancer Res Clin Oncol. 2018; 144(8):1591-9.

2. Zhang Z, Chen ML, Gu XL, Liu MH, Zhao WW, Cheng WW. Palliative Chemotherapy Near the End of Life in Oncology Patients. Am J Hosp Palliat Care. 2018; 35(9):1215-20.

3. Bao Y, Maciejewski RC, Garrido MM, Shah MA, Maciejewski PK, Prigerson HG. Chemotherapy Use, End-of-Life Care, and Costs of Care Among Patients Diagnosed with Stage IV Pancreatic Cancer. J Pain Symptom Manage. 2018; 55(4):1113-21 e3.

4. Schulkes KJG, van Walree IC, van Elden LJR, van den Bos F, van Huis-Tanja L, Lammers JJ, et al. Chemotherapy and healthcare utilization near the end of life in patients with cancer. Eur $\mathrm{J}$ Cancer Care (Engl). 2018; 27(2): e12796.

5. Norton SA, Wittink MN, Duberstein PR, Prigerson HG, Stanek S, Epstein RM. Family caregiver descriptions of stopping chemotherapy and end-of-life transitions. Support Care Cancer. 2018.

6. Matsuyama R, Reddy S, Smith TJ. Why do patients choose chemotherapy near the end of life? A review of the perspective of those facing death from cancer. J Clin Oncol. 2006; 24(21):3490-6.

7. Rochigneux P, Raoul JL, Beaussant Y, Aubry R, Goldwasser F, Tournigand C, et al. Use of chemotherapy near the end of life: what factors matter? Ann Oncol. 2017; 28(4):809-17.

8. Low D, Merkel EC, Menon M, Lyman GH, Ddungu H, Namukwaya E, et al. Chemotherapy Use at the End of Life in Uganda. J Glob Oncol. 2017; 3(6):711-9. 
9. Hui D, Park M, Liu D, Paiva CE, Suh SY, Morita T, et al. Clinician prediction of survival versus the Palliative Prognostic Score: Which approach is more accurate? Eur J Cancer. 2016; 64:89-95.

10. Wu CK, Kao SJ, Lai HC. Targeted Therapy and Immunotherapy Lead to Rapid Regression of Advanced Non-Small Cell Lung Cancer with Multiple Driver Mutations. J Thorac Oncol. 2018; 13(6): e103-e5.

11. Mathew A, Achkar T, Abberbock S, Sandhu GS, Jacob ME, Villgran VD, et al. Prevalence and determinants of end-of-life chemotherapy use in patients with metastatic breast cancer. Breast J. 2017; 23(6):718-22.

12. Liu TW, Chang WC, Wang HM, Chen JS, Koong SL, Hsiao SC, et al. Use of chemotherapy at the end of life among Taiwanese cancer decedents, 2001-2006. Acta Oncol. 2012; 51(4):505-11.

13. Adam H, Hug S, Bosshard G. Chemotherapy near the end of life: a retrospective single-center analysis of patients' charts. BMC Palliat Care. 2014; 13:26.

14. Mohammed AA, Al-Zahrani AS, Ghanem HM, Farooq MU, El Saify AM, El-Khatib HM. End-of-life palliative chemotherapy: where do we stand? J Egypt Natl Canc Inst. 2015; 27(1):35-9.

15. Prigerson HG, Bao Y, Shah MA, Paulk ME, LeBlanc TW, Schneider BJ, et al. Chemotherapy Use, Performance Status, and Quality of Life at the End of Life. JAMA Oncol. 2015; 1(6):778-84.

16. McCarthy M. Chemotherapy does not improve quality of life in cancer patients at end of life, US study finds. BMJ. 2015; 351:h4139.

17. Ho TH, Barbera L, Saskin R, Lu H, Neville BA, Earle CC. Trends in the aggressiveness of end-of-life cancer care in the universal health care system of Ontario, Canada. J Clin Oncol. 2011; 29(12):158791.

18. Temel JS, Greer JA, Muzikansky A, Gallagher ER, Admane S, Jackson VA, et al. Early palliative care for patients with metastatic non-small-cell lung cancer. N Engl J Med. 2010; 363(8):733-42.

\section{Tables:}

Due to technical limitations, table 1 and 2 PDFs are only available as a download in the Supplemental Files section.

\section{Supplementary Files}

This is a list of supplementary files associated with this preprint. Click to download.

- Table1chemolast4weeks.pdf

- Table2chemolas4weeksedited.pdf 